\title{
Patient-Specific Time-Varying Association between Spatial and Temporal Variability in Repolarization and High Sensitivity Troponin I
}

\author{
Larisa G. Tereshchenko ${ }^{1,2}$, Albert Feeny ${ }^{2}$ \\ ${ }^{1}$ Knight Cardiovascular Institute, Oregon Health and Science University, Portland, Oregon, USA, \\ ${ }^{2}$ Johns Hopkins University, Baltimore, Maryland, USA.
}

\begin{abstract}
We hypothesized that the patient-specific time-varying changes in the spatial and temporal variability in cardiac repolarization (quantified by spatial TT' angle), and in myocardial injury (measured by high sensitivity troponin I, hsTnI), are independently associated with each other.

Spatial TT' angle on resting 12-lead ECG (transformed to vectorcardiogram) and hsTnI were measured simultaneously every 3 hours during a 12-hour observation period in a prospective cohort of emergency department patients ( $n=379$; age $57.8 \pm 13.2 y$; $54 \%$ female, $64 \%$ black), diagnosed with acute coronary syndrome (ACS; $n=28$ ), acute decompensated heart failure (ADHF; $n=35)$, or an acute non-cardiac condition $(n=316)$.

High (above median) HsTnI in ACS was characterized by significantly larger $T T$ ' angle (12 \pm 8 vs $5 \pm 2$ degrees; $P=0.01) 12$ hours after admission, but not earlier. In adjusted multinomial logit model, spatial TT' angle was associated with ADHF (Relative Risk Ratio 6.24 (95\%CI 1.32-29.57; $P=0.021$ ), but not ACS. After full adjustment for confounders in random-effect linear regression, a 10fold increase in hsTnI in a specific study participant was associated with 1.05 (95\% CI $0.19-1.92)$ degrees increase in spatial TT' angle. Longitudinal association of hsTnI and TT' angle was especially prominent in patients with acute non-cardiac conditions, but not in ACS or ADHF.
\end{abstract}

\section{Introduction}

Increased spatial and temporal variability in cardiac repolarization, as quantified by an angle between two consecutive sinus beats' spatial T vectors, averaged across 10 seconds, has been shown independently associated with sudden cardiac death (SCD) in the general population [1], as well as with ventricular tachyarrhythmias in cardiomyopathy patients with implanted primary prevention cardioverter-defibrillators [2].

Experimental studies [3] showed that the magnitude of beat-to-beat variability in action potential duration due to stochastic gating in calcium, sodium, and potassium ion channels is very small, and would be unlikely detected on the surface ECG [3] unless amplified by cell-to-cell uncoupling. Thus, measured on the surface ECG spatial and temporal variability in cardiac repolarization could be considered as a test for cell-to-cell coupling.

Acute myocardial injury affects cardiac myocytes coupling. Fast and massive cell-to-cell uncoupling in response to acute myocardial injury is adaptive as it prevents the spread of injury mediators from severely to less affected areas, and therefore decreases damage to the heart [4]. However, uncoupling of myocytes slows ventricular conduction and leads to ventricular tachyarrhythmia [5]. In opposite, slight and delayed cellto-cell uncoupling in response to acute myocardial injury [6] would be unlikely capable of enhancing repolarization variability and increasing risk of ventricular tachyarrhythmias, but instead, could lead to large transmural myocardial infarction with increased risk of myocardial rupture, cardiogenic shock, asystole, and pulseless electrical activity.

Acute coronary syndrome (ACS) and acute decompensated heart failure (ADHF) are characterized by acute myocardial injury, albeit due to two different mechanisms. Association between spatial and temporal variability in cardiac repolarization and the degree of myocardial injury, quantified by the level of highsensitivity troponin I (hsTnI), has not been previously studied. We addressed this knowledge gap in our study.

\subsection{Hypothesis}

We hypothesized that the patient-specific time-varying changes in the spatial and temporal variability in cardiac repolarization (quantified by spatial TT' angle), and in myocardial injury (measured by hsTnI), are independently associated with each other.

\section{Methods}

This is an ancillary study of a prospective observational cohort study [7-9] of emergency department (ED) patients evaluated for ACS at the Johns Hopkins Hospital (JHH). 
The study was approved by the Institutional Review Board, and all participants provided written informed consent.

\subsection{Patient population}

Inclusion and exclusion criteria of the JHH ED prospective cohort study have been previously described [7-9]. Briefly, the original cohort [7] enrolled consecutive patients with suspected ACS of age 25 or older, who presented to the JHH ED with a non-diagnostic initial ECG. The final clinical diagnosis was adjudicated by an independent end-point adjudication committee. In this ancillary study, we included participants with (1) available serial digital resting ECG and hsTnI measurements performed every 3 hours during a period of observation in the ED, and (2) a final diagnosis of ACS, ADHF, or noncardiac condition. Participants with ECGs in rhythm other than sinus were excluded.

\subsection{Spatial TT' angle measurement}

Digital 12-lead ECG was transformed into orthogonal ECG using the inverse Dower transformation. After removal of respiration, spatial TT' angle was measured for each pair of consecutive sinus beats as an angle between two consecutive sinus beats' spatial $\mathrm{T}$ vectors, as previously described $[1,2,10]$. The mean spatial TT' angle was then calculated by averaging all TT' angles across 10 seconds.

\subsection{Statistical analyses}

Pearson's chi-square test was used to compare categorical variables. HsTnI and TT' angle values were log-10-transformed to normalize their distribution, prior to inclusion in regression models. ANOVA with Bonferroni correction for multiple comparisons was used to compare continuous variables across three clinical groups.

A multinomial logit model adjusted by age, sex, race, prevalent cardiovascular disease (myocardial infarction, history of $\mathrm{HF}$ and revascularization), and risk factors (diabetes, smoking, hypercholesterolemia, hypertension, cocaine use) was used to characterize differences between ACS, ADHF and non-cardiac conditions (reference) at ED admission, $6^{\text {th }}$, and $12^{\text {th }}$ hours of observation.

In order to determine whether the patient-specific timevarying changes in the spatial TT' angle are associated with the patient-specific time-varying hsTnI changes during 12-hour observation in the ED, we conducted a generalized least squares random-effects linear regression analysis. Patient-specific time-varying hsTnI served as a predictor. Patient-specific time-varying TT' angle served as an outcome. We performed the Hausman test to choose between the random-effect estimator (assuming that the unobserved time-invariant random component is unrelated to the predictors) and fixed-effect estimator (allowing the unobserved random component to be related to the predictors). Model 1 was adjusted for demographic characteristics (age, sex, and race). Model 2 included all variables in the Model 1, as well as prevalent cardiovascular disease (prior myocardial infarction, prior revascularization procedure (coronary artery bypass grafting or percutaneous transluminal coronary angioplasty, and HF). Model 3 included all the Model 2 variables, and known risk factors of cardiovascular disease (smoking, diabetes, hypertension, hypercholesterolemia), cocaine use, and left bundle branch block.

Statistical analysis was performed using STATA 14 (StataCorp LP, College Station, TX).

\section{Results}

\subsection{Study population}

The study population included 379 participants (age 57.8 $\pm 13.2 y$; $54 \%$ female, $64 \%$ black), diagnosed with ACS $(n=28), \operatorname{ADHF}(n=35)$, or a non-cardiac condition $(n=316)$. Clinical characteristics of study groups have been previously described [8].

Overall mean spatial TT' angle was 9.0 $48.1^{\circ}$. During the 12-hour observation period in ED, TT' angle was measured on average 3.2 times, every 3 hours, and demonstrated fluctuations over time in 346 (91\%) of study participants (Table 1). There was no statistically significant difference in baseline spatial TT' angle across study groups (Table 1). On average, there were no statistically significant changes in spatial TT' angle over the 12-hour observation period in ED. Dynamic changes in hsTnI in this study participants have been previously described [8].

Table 1. Spatial TT’ angle in Study Participants.

\begin{tabular}{|c|c|c|c|c|}
\hline & $\begin{array}{l}\text { ACS } \\
(n=28)\end{array}$ & $\begin{array}{l}\text { ADHF } \\
(n=35)\end{array}$ & $\begin{array}{l}\text { Non- } \\
\text { cardiac } \\
(n=316)\end{array}$ & $\begin{array}{l}\text { ANOV } \\
\mathrm{A} / \chi^{2} \mathrm{P}\end{array}$ \\
\hline $\begin{array}{l}\text { Baseline TT' } \\
\text { angle (SD), }^{\circ}\end{array}$ & $\begin{array}{l}8.8 \\
(7.6)\end{array}$ & $\begin{array}{l}11.6 \\
(7.7)\end{array}$ & 8.5 (8.3) & 0.163 \\
\hline $\begin{array}{l}\text { TT' angle only } \\
\text { up, n (\%) }\end{array}$ & $1(4)$ & 0 & $18(6)$ & \\
\hline $\begin{array}{l}\text { TT' angle up \& } \\
\text { down, n (\%) }\end{array}$ & 27 (96) & 35 (100) & $284(90)$ & 0.249 \\
\hline $\begin{array}{l}\text { TT’ angle only } \\
\text { down, n (\%) }\end{array}$ & 0 & 0 & $14(4)$ & \\
\hline
\end{tabular}

\subsection{Association of TT' angle with ACS and ADHF vs. non-cardiac condition}

In multinomial logit models, adjusted for 
demographics (age, sex, race), prevalent cardiovascular disease (prior myocardial infarction, history of revascularization, HF), and risk factors (hypertension, diabetes, smoking, cocaine use) spatial TT' angle was associated with ADHF (Table 2), but not ACS (Table 3). Non-cardiac condition served as a reference in the models.

Table 2. Relative Risk Ratio of TT' angle being associated with ADHF in adjusted Multinomial Logit Model.

\begin{tabular}{cccc}
\hline Hour in ED & RRR & 95\% Confidence Interval & P-value \\
\hline $1^{\text {st }}$ & 6.24 & $1.32-29.57$ & 0.021 \\
\hline $6^{\text {th }}$ & 13.0 & $1.24-136.69$ & 0.032 \\
\hline $12^{\text {th }}$ & 6.68 & $0.27-164.45$ & 0.245 \\
\hline
\end{tabular}

Table 3. Relative Risk Ratio (RRR) of TT' angle being associated with ACS in adjusted Multinomial Logit Model.

\begin{tabular}{cccc}
\hline Hour in ED & RRR & 95\% Confidence Interval & P-value \\
\hline $1^{\text {st }}$ & 0.84 & $0.17-4.19$ & 0.831 \\
\hline $6^{\text {th }}$ & 1.94 & $0.09-41.04$ & 0.672 \\
\hline $12^{\text {th }}$ & 22.53 & $0.29-1,716.43$ & 0.159 \\
\hline
\end{tabular}

Elevated (above median) hsTnI in ACS group was characterized by significantly larger TT' angle (12 \pm 8 vs $5 \pm 2$ degrees; $\mathrm{P}=0.01$ ) 12 hours after $\mathrm{ED}$ admission, but not earlier. Of note, patients with high (above $99^{\text {th }}$ percentile) hsTnI in ACS $12^{\text {th }}$ hours after admission had a mean TT' angle 10.0 (95\%CI 6.3-13.8) degrees.

In the ADHF group, high (above $99^{\text {th }}$ percentile) hsTnI was associated with significantly larger TT' angle (10.7 \pm 5.2 vs. $3.6 \pm 1.4$ degrees; $\mathrm{P}=0.004)$ at any time in $\mathrm{ED}$.

There was no difference in TT' angle in patients with acute non-cardiac condition and hsTnI above vs. below $99^{\text {th }}$ percentile $(8.5 \pm 8.5$ vs. $8.9 \pm 7.1$ degrees; $\mathrm{P}=0.743)$.

\subsection{Patient-Specific dynamic changes in spatial TT' angle}

The Hausman test indicated that the random-effects estimators were the most consistent and efficient for patient-specific analysis of dynamic changes in TT' angle. Thus, random-effects estimators are reported for all patient-specific models in this study (Tables 4-7). We observed a significant association between dynamic changes in spatial TT' angle and hsTnI. Dynamic changes in TT' angle and hsTnI were concordant. Overall, a 10-fold increase in hsTnI in a specific study participant was associated with an approximately 1-degree increase in spatial TT' angle (Table 4) in the same participant. Accordingly, a 10-fold decrease in hsTnI in another patient was associated with about 1-degree decrease in TT' angle in that patient. Adjustment for prevalent and subclinical cardiovascular disease slightly attenuated the association.
Subgroups analyses (Tables 5-7) showed that direct association between dynamic changes in spatial TT' angle was present in patients with non-cardiac conditions only (Table 7), but not in ACS (Table 5) or ADHF (Table 6) patients.

Table 4. Patient-Specific Time-Varying Difference (95\%CI) in TT' angle by hsTnI in all study participants

\begin{tabular}{ccc}
\hline Model & Per 10-fold hsTnI $(95 \%)$ increase & P-value \\
\hline 1 & $1.20(0.38-2.02)$ & 0.004 \\
\hline 2 & $1.02(0.16-1.87)$ & 0.020 \\
\hline 3 & $1.05(0.19-1.92)$ & 0.017 \\
\hline
\end{tabular}

Table 5. Patient-Specific Time-Varying Difference (95\%CI) in TT' angle by hsTnI in ACS

\begin{tabular}{ccc}
\hline Model & Per 10-fold hsTnI $(95 \%)$ increase & P-value \\
\hline 1 & $-0.12(-2.37-2.13)$ & 0.918 \\
\hline 2 & $0.34(-2.53-3.21)$ & 0.817 \\
\hline 3 & $1.26(-1.79-4.31)$ & 0.418 \\
\hline
\end{tabular}

Table 6. Patient-Specific Time-Varying Difference (95\%CI) in TT' angle by hsTnI in ADHF

\begin{tabular}{ccc}
\hline Model & Per 10-fold hsTnI $(95 \%)$ increase & P-value \\
\hline 1 & $1.05(-5.40-7.51)$ & 0.750 \\
\hline 2 & $-0.24(-6.72-6.25)$ & 0.943 \\
\hline 3 & $-1.11(-7.37-5.16)$ & 0.729 \\
\hline
\end{tabular}

Table 7. Patient-Specific Time-Varying Difference (95\%CI) in TT' angle by hsTnI in non-cardiac condition

\begin{tabular}{ccc}
\hline Model & Per 10-fold hsTnI $(95 \%)$ increase & P-value \\
\hline 1 & $1.63(0.61-2.65)$ & 0.002 \\
\hline 2 & $1.63(0.57-2.69)$ & 0.003 \\
\hline 3 & $1.53(0.45-2.61)$ & 0.006 \\
\hline
\end{tabular}

\section{Discussion}

The main finding of this study was an observation of a significant independent direct patient-specific timevarying association between the surface ECG measure of spatial and temporal variability in cardiac repolarization spatial TT' angle and hsTnI. Association between spatial TT' angle and hsTnI was significant in patients with acute non-cardiac conditions, but not in ACS or ADHF. ADHF patients were characterized by elevated baseline TT' angle. ACS patients were characterized by at least 12-hours delay in elevation of TT' angle. Taken together, results of our study support the hypothesis of the spatial and temporal variability of cardiac repolarization essentially being a measure of the cardiac myocytes uncoupling.

The effect of uncoupling was especially prominent in patients with non-cardiac conditions, with presumably 
normally coupled myocytes at baseline. Consistently with our previous study [1], the average baseline level of TT' angle in this group was within the range of normal general population reference level $\left(<9.57^{\circ}\right)$. Injury of the myocardium in an acute non-cardiac condition is secondary and minor (detected only by hsTnI). New minor injury of cardiomyocytes (detected by elevating hsTnI) was associated with reversible cell-to-cell uncoupling (manifested by concordantly increasing TT' angle). Accordingly, dynamically decreasing level of hsTnI was associated with improving cell-to-cell coupling and concordantly decreasing TT' angle. Importantly, observed concordant changes in hsTnI and TT' angle were patientspecific, but not group-averaged.

The baseline level of TT' angle in ACS at the time of ED admission did not differ from that in a reference healthy general population, which suggests normal cell-tocell coupling. Importantly, 12 hours later, a high level of hsTnI was characterized by at least a twice as high level of TT' angle. Thus, there was a lag in time between ACS clinical manifestation (resulted in ED admission) and uncoupling of cardiomyocytes (manifested by TT' angle increase 12 hours after ED admission). Results of our study suggest that there is large individual variability in the timing and degree of uncoupling, as we did not observe a statistically significant patient-specific association between hsTnI and TT' angle in ACS patients.

The presence of increased TT' angle at baseline was associated with significantly increased relative risk of having ADHF. Doubling of TT' angle was associated with increased relative risk of ADHF by at least 6-fold. Averaged TT' angle values in the ADHF group were consistent with abnormal TT' angle values $\left(\geq 9.57^{\circ}\right)$, associated with increased risk of sudden cardiac death in the general population [1]. Thus, ADHF patients at baseline have already had uncoupled myocytes, and the acute myocardial injury was not able to affect it further.

Limitations of the study must be considered. This study was an ancillary retrospective analysis of a prospective cohort study. The study was not powered for subgroups (ACS and ADHF) analyses. The sufficiently powered prospective study is needed to confirm our findings. However, use of the hsTnI assay is an important strength of the study, as hsTnI assay is the most sensitive; it detects values in $96 \%$ of normal subjects.

\section{Conclusion}

Patient-specific time-varying changes in the surface ECG measure of spatial and temporal variability in cardiac repolarization TT' angle are independently and directly associated with patient-specific time-varying changes in hsTnI of patients diagnosed with non-cardiac conditions, likely reflecting the extent of cardiac myocytes uncoupling in the previously unaffected myocardium.

\section{Acknowledgements}

Authors thank JHH ED cohort investigators Frederick K. Korley, MD, PhD, Erica Shelton, M.D, M.P.H., Thomas Metkus, M.D, Andrew Stolbach, M.D, Ernest Mavunga, M.D., M.P.H, and Shannon Putman, M.D.

This work was partially supported by the NIH R01HL118277 (LGT).

\section{References}

[1] Waks JW, Soliman EZ, Henrikson CA, Sotoodehnia N, Han L, Agarwal SK, et al. Beat-to-beat spatiotemporal variability in the $T$ vector is associated with sudden cardiac death in participants without left ventricular hypertrophy: the Atherosclerosis Risk in Communities (ARIC) Study. J Am Heart Assoc. 2015;4(1):e001357.

[2] Tereshchenko LG. Repolarization Lability Measured by Spatial TT' Angle. Computing in cardiology. 2014;41:1814.

[3] Pueyo E, Corrias A, Virag L, Jost N, Szel T, Varro A, et al. A multiscale investigation of repolarization variability and its role in cardiac arrhythmogenesis. BiophysJ. 2011;101(12):2892-902.

[4] Kanno S, Kovacs A, Yamada KA, Saffitz JE. Connexin43 as a determinant of myocardial infarct size following coronary occlusion in mice. $\mathrm{J}$ Am Coll Cardiol. 2003;41(4):681-6.

[5] Lerner DL, Yamada KA, Schuessler RB, Saffitz JE. Accelerated onset and increased incidence of ventricular arrhythmias induced by ischemia in Cx43-deficient mice. Circulation. 2000;101(5):547-52.

[6] Zhuang J, Yamada KA, Saffitz JE, Kleber AG. Pulsatile stretch remodels cell-to-cell communication in cultured myocytes. CircRes. 2000;87(4):316-22.

[7] Korley FK, Schulman SP, Sokoll LJ, DeFilippis AP, Stolbach AI, Bayram JD, et al. Troponin elevations only detected with a high-sensitivity assay: clinical correlations and prognostic significance. Academic emergency medicine : official journal of the Society for Academic Emergency Medicine. 2014;21(7):727-35.

[8] Tereshchenko LG, Feeny A, Shelton E, Metkus T, Stolbach A, Mavunga E, et al. Dynamic Changes in High-Sensitivity Cardiac Troponin I Are Associated with Dynamic Changes in Sum Absolute QRST Integral on Surface Electrocardiogram in Acute Decompensated Heart Failure. Ann Noninvasive Electrocardiol. 2016.

[9] Tereshchenko LG, Gatz D, Feeny A, Korley FK. Automated analysis of the 12-lead ECG in the emergency department: association between high-sensitivity cardiac troponin I and the cardiac electrical biomarker. Critical pathways in cardiology. 2014;13(1):25-8.

[10. Sur S, Han L, Tereshchenko LG. Comparison of sum absolute QRST integral, and temporal variability in depolarization and repolarization, measured by dynamic vectorcardiography approach, in healthy men and women. PLoS One. 2013;8(2):e57175.

Address for correspondence.

Larisa G. Tereshchenko, MD, PhD, 3181 SW Sam Jackson Park Rd; Portland, OR, 97239. E-mail address: tereshch@ohsu.edu 